\title{
Hypertonie und Schlaganfall
}

A nhand epidemiologischer, seit 1981 kontinuierlich erhobener Daten aus dem „Oxford Community Stroke Project" und der "Oxford Vascular Study“ zeigte Peter Rothwell in seiner unlängst im Lancet publizierten Arbeit, dass heute signifikant weniger Schlaganfälle aufgrund intrazerebraler Blutungen $(>50 \%)$ und schwere Schlaganfälle mit ungünstigem Ausgang (Tod/ schweres Defizit) auftreten als noch vor 20 Jahren. Laut der sorgfältigen Analyse der mit diesen Ereignissen assoziierten Risikofaktoren ging zum einen der Anteil an Rauchern zurück, zum anderen werden mehr oder minder kontinuierliche Medikationen mit Antihypertensiva, Lipidsenkern und Plättchenantagonisten im Vorfeld eines Schlaganfalls für diesen Erfolg angeführt.

Liest man unsere Beiträge in dieser Ausgabe des klinikarzt zum Blutdruckmanagement beim akuten Schlaganfall und in der Nachsorge, so kontrastiert der Mangel an evidenzbasierten Daten zur Sekundärprophylaxe mit diesen Befunden zur Primärprävention aus angelsächsischen, traditionell epidemiologisch-wissenschaftlich stark engagierten Ländern: Scheinbar einfache Fragen bleiben weit gehend unbeantwortet: Sollen die in der Akutphase eines Schlaganfalls gemessenen hohen Blutdruckwerte medikamentös gesenkt werden - wenn ja, ab wann und in welchem Umfang? Sollen erhöhte oder auch im Normbereich liegende Blutdruckwerte nach einer transitorischen ischämischen Attacke oder einem Schlaganfall langfristig gesenkt werden - wenn ja, in welchem Ausmaß und mit welchen Substanzen?

Während die medizinische Grundlagenforschung schon wegen der mangelnden Eignung ihrer experimentellen Modelle auch nicht annäherungsweise den Versuch unternommen hat, solchen klinisch wichtigen Fragen nachzugehen, sind klinische Studien, soweit sie denn existieren, entweder überholt, vom Design inadäquat oder nicht aussagekräftig angelegt. Dies liegt wesentlich an der mangelnden Förderung klinischer Forschungsprojekte, gerade auch in Deutschland. Neben Studien, die durch das einseitige Interesse an bestimmten Substanzen seitens der pharmazeutischen Industrie geprägt sind, ist es nicht gelungen, randomisierte prospektive klinische Therapiestudien, die aus wissenschaftlicher Sicht sinnvoll wären, zum Gegenstand klinischer Forschung zu machen. Im Gegensatz zu den angelsächsischen Ländern, wo das „National Institute of Health“ (NIH) und das „Medical Research Council“ (MRC) solche Studien massiv finanziell unterstützen oder wenigstens auf eine hohe Qualität achten, haben wichtige Therapiestudien hierzulande keine Chance: Weder die Deutsche Forschungsgemeinschaft (DFG) noch das Bundesministerium für Bildung und Forschung (BMBF) sind daran interessiert.

Erst vor wenigen Monaten hat sich die DFG auf massives Drängen hin bereit erklärt, einen minimalen Anteil ihrer Mittel zur Evaluation der dann in der Regel wegen mangelnder Finanzkraft abgelehnten Anträge zu klinischen Studien zu benutzen: Zwar fordert der Wissenschaftsrat, Strukturen und Organisationen an den medizinischen Fakultäten zu verändern, um die klinische Forschung zu stärken, gleichzeitig empfiehlt er aber den Universitätskliniken, ihre Aufgaben in der Krankenversorgung drastisch zu reduzieren. Für Volkskrankheiten wie Schlaganfall und Herzinfarkt ist es jedoch unerlässlich, einen ausreichenden Zugang zu großen Patientenzahlen zu behalten, um aus einem großen Screeningpool geeignete Rekrutierungen für randomisierte klinische Studien zu schaffen und diese dann über Jahre zu betreuen.

Die neuen Gesetze bzw. Richtlinien der zwölften Novelle des Arzneimittelgesetzes (AMG) gestalten bislang vom Standort initiierte klinische Prüfungen bzw. Studien so kompliziert, dass selbst mit einem universitären Monitoring bislang erfolgreiche Phase-I/II-Studien (von Phase III gar nicht zu reden), an exorbitanten Kosten und hohem Dokumentationsumfang scheitern werden. Hinzu kommt neben allen bürokratischen Erschwernissen ein wachsendes Desinteresse der Pharmaindustrie an deutschen oder europäischen Kooperationspartnern, sie verlagert ihre Aktivitäten ins angelsächsische Ausland.

Wie soll aber evidenzbasierte Medizin funktionieren, wenn der Datenstrom aus der klinischen Forschung versiegt? Neue wissenschaftliche Konzepte gibt es reichlich: Zum Beispiel existiert schon seit mehreren Jahren die Idee, durch eine „Polypille“ das kardio-zerebro-vaskuläre Risiko möglicherweise höchst wirksam und kosteneffizient zu senken - allein die Umsetzung verharrt mangels gescheiter, entschlossener Forschungsförderung weiter in trauriger Unbeweglichkeit! So haben sich die pathophysiologischen Konzepte und die methodischen Strategien zur Beeinflussung erkannter Krankheitsursachen zwar im Laufe der Jahrhunderte erweitert, therapeutisch verharren wir aber weiterhin im 18. bzw. 19. Jahrhundert, wenn sich an diesen Strukturen und Forschungsgegebenheiten nichts ändert.

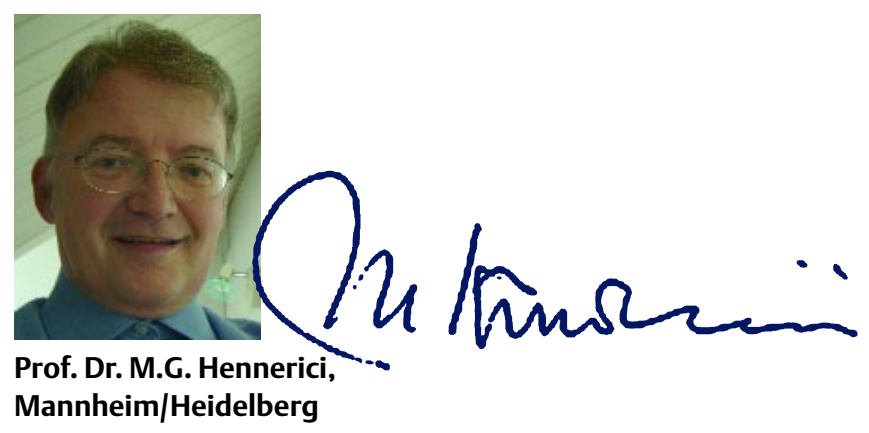

